\title{
Porphyromonas gingivalis Biofilm Formation on Implant Surface Treated with Nanoselenium
}

\author{
Mohamed Assadawy ${ }^{1, *}$ and Eman Helmy ${ }^{2}$ \\ ${ }^{1}$ Department of Oral Medicine, Periodontology, Oral Diagnosis and Oral Radiology, Faculty of dental medicine, Al-Azhar University, Cairo, Egypt \\ ${ }^{2}$ Regional Center for Mycology and Biotechnology, Al-Azhar University, Cairo, Egypt
}

*Corresponding author: Mohamed Assadawy, Lecturer, Department of Oral Medicine, Periodontology, Oral Diagnosis and Oral Radiology, Faculty of dental medicine, Al-Azhar University, PO 11825, Cairo, Egypt, E-mail: Drmohamed_i@yahoo.com

Received: 05 May, 2021 | Accepted: 25 May, 2021 | Published: 01 Jun, 2021

Citation: Assadawy M, Helmy E (2021) Porphyromonas gingivalis Biofilm Formation on Implant Surface Treated with Nanoselenium. Int J Dent Oral Health 7(5): dx.doi.org/10.16966/2378-7090.366

Copyright: @ 2021 Assadawy M, et al. This is an open-access article distributed under the terms of the Creative Commons Attribution License, which permits unrestricted use, distribution, and reproduction in any medium, provided the original author and source are credited.

\section{Abstract}

Introduction: Biofilm formation on implants is the primary factor for implant loss. Porphyromonas gingivalis is a highly virulent pathogen that contributes to the development of periodontal disease and implant failure.

Objectives: The goals of this study are to investigate the formation of $P$. gingivalis biofilms on nanoselenium coated implants in vitro and the potential use of nanoselenium for peri-implantitis treatment.

Materials and methods: Porphyromonas gingivalis ATCC 33277 was cultured to obtain an in vitro mature biofilm on the surface of the Hexacone implant system. The fixture was added into an Eppendorf tube and placed in a sterile air laminar flow cabinet. An automatic machine learning utility was used to calculate the biofilm size on the implant surface from SEM images, and the Trainable Weka Segmentation plugin in Fiji software was employed.

Results: The SeNPs affected the $P$. gingivalis biofilm (the effect size was $80.17 \%$ ), and the difference was highly significant ( $p 0.000$ ).

Conclusion: The use of SeNPs as dental implant coatings presented promising anti-P. gingivalis biofilm activity.

Clinical relevance: The development of a dental implant surface treatment with efficient antibacterial properties, especially against the most virulent pathogens, has not yet been established.

Principal findings: Nanoselenium particles as an implant surface coating prevented Porphyromonas gingivalis biofilm formation to a striking extent.

Practical implication: Nanoparticles could provide a novel state-of-the-art therapeutic approach for Porphyromonas gingivalis (P. gingivalis biofilm on dental implants).

Keywords: Peri-implantitis; Periodontal disease; Nanoselenium Imaging; Porphyromonas gingivalis; Bacterial biofilm

Abbreviations: ATCC 33277: Porphyromonas gingivalis ATCC 33277 DNA, complete genome; P. gingivalis: Porphyromonas gingivalis; T. denticola: Treponema denticola; SeNP: Selenium Nanoparticles; SEM: Scanning Electron Microscopy; WEKA: Waikato Environment for Knowledge AnalysisWEKA is a collection of machine learning algorithms for data mining tasks. WEKA contains tools for data preprocessing, classification, regression, clustering, association rules, and visualization; MIC: Minimum Inhibitory Concentration

\section{Introduction}

Biofilm formation on implants is a primary factor in the periimplant disease. Porphyromonas gingivalis is the main causative pathogen in periodontitis. This microorganism is also a risk factor for various systemic diseases, such as certain disorders, diabetes, and pulmonary infection. The features of microbes in a biofilm can deviate significantly from that of an equivalent organism under planktonic conditions in terms of the rate of growth and gene transcription. Clinically, biofilms form on the skin, oral mucosa and teeth sometimes cause chronic infection of dental implants [1].
Management of biofilm-related infections brings great challenges in oral implantology because the structure and composition of the biofilm itself offers protection against antimicrobial agents, and regular mechanical biofilm disruption is required to enable surface disinfection. Medications that may remove such biofilms are needed for clinical use. Dental implants have become the first choice for patients seeking dental restorations. However, implant mucositis and subsequent peri-implantitis impose a great risk for implant quality and patient comfort. Additionally, bone resorption and even implant loosening account for nearly $30 \%$ of total implant failure cases [2]. 
Significant data have confirmed that biofilm formation on the implant neck is responsible for implant mucositis [3].

$P$. gingivalis has been confirmed as a critical pathogen in periimplantitis, periodontitis represented by inflammation of periimplant soft and hard tissues. This condition may result in dental implant failure $[4,5]$, and a considerably high prevalence of periimplantitis (20\% to 56\%) has been reported [6]. Biofilms are complex communities of microorganisms that are adherent to each other and/ or to a surface and are encapsulated within a self-produced matrix [7]. These organized communities represent a major implant risk because of their invasion and evasion of host defense mechanisms and their decreased susceptibility to antimicrobials [8]. Biofilm-mediated resistance is due to impaired penetration of antimicrobials, upregulation of drug resistance genes, and downregulation of metabolic activity of cells included within the biofilm $[9,10]$. The pathogenicity of $P$. gingivalis is expressed by an arsenal of virulence factors connected with tissue colonization and damage and hinders host defense mechanisms [11]. Nutritional interactions are described to play a role regarding the coexistence of $P$. gingivalis and $T$. denticola. $P$. gingivalis provides isobutyric acid, which promotes the growth of T. denticola, while T. denticola produces succinate, which enhances the growth of $P$. gingivalis; these interactions might explain the finding that $P$. gingivalis and T. denticola exhibit enhanced planktonic and biofilm growth once they are cultured together compared to monospecies growth $[12,13]$. Antibacterial agents that possess activity against $P$. gingivalis include quorum sensing inhibitors, antimicrobial peptides, and natural sources such as capsaicin from Capsicum plants (chili peppers) $\mu \mathrm{g} / \mathrm{mL}$ [14]. Selenium, an important element, is critical for health. In humans, selenium is necessary for the synthesis of 25 selenoproteins. The beneficial effects of selenium on the risk of various cancers (lung, liver, colorectal, prostate, esophageal, gastric cardia, thyroid and bladder) have been confirmed [15]. SeNPs have been studied for certain medical uses and as a possible substance for orthopedic implants [16]. The power of selenium compounds as antibiofilm and anti-inflammatory agents has been confirmed. Nanostructured materials improve osteoblast functions (such as adhesion [17], proliferation, synthesis of certain bone proteins, and deposition of calcium-containing minerals) and encourage adequate osteointegration because of the maximized area and roughness $[18,19]$. In contrast, tailoring the surface of titanium dental implants with antibacterial agents is a critical aim for implantologists and researchers. Antimicrobial coatings inhibit the infection risks of implants, which are the foremost common explanation for reverse surgery. The antibacterial actions of NPs can be classified as (1) damaging the cell membrane, causing cell lysis; (2) disrupting protein synthesis; and (3) preventing DNA replication $[20,21]$. Many different methods are used to assess biofilms. Biological techniques include the following: semi-quantitative staining, measurements of dried biomass, protein or DNA quantification, and assessments of residual viable organisms. Each method has advantages and deficiencies, but all of them provide only indirect values of the removal efficiency and are susceptible to operator variability [22-24]. Standard optical microscopy, confocal laser scanning microscopy and epifluorescence microscopy (EM) are reliable tools for biofilm analysis. Additionally, scanning electron microscopy (SEM) is a proper instrument not only for intimately viewing the substratum morphology but also for observing bacterial attachment and biofilm formation on abiotic surfaces. Indeed, SEM has been useful within the event of antibiofilm materials for biomedical applications [25,26]. Scanning electron microscopy (SEM) has been used extensively for qualitative observation of biofilms because of its high resolution and is typically applied in conjunction with biological assays on bacterial biofilms [27]; advanced segmentation techniques such as semisupervised machine learning methods are also typically prescribed [28].

\section{Material and Methods}

The minimum inhibitory concentration of SeNPs was tested by the microtiter broth dilution method as described by Khiralla GM, et al., [29]. The concentrations of SeNPs were $0,10,15,20,25$ and $30 \mu \mathrm{g} / \mathrm{mL}$. The MIC90 was set as the lowest concentration of SeNPs that inhibited $90 \%$ of the growth compared with the positive control. All tests were administered in triplicate $(\mathrm{n}=3)$, and the results were averaged [30].

Nano preparation an actively growing culture was used to prepare subcultures on potato dextrose agar slants, and after 72 hours of incubation at $28^{\circ} \mathrm{C}$, the slant was used as the starting material for titanium nanoparticle synthesis. The fungus was cultivated in a $250 \mathrm{~mL}$ flask containing $100 \mathrm{~mL}$ of modified malt extract-peptone (MGYP) medium. The $\mathrm{pH}$ of the medium was set to six, and a rotary shaker was operated at $150 \mathrm{rpm}$ and $28^{\circ} \mathrm{C}$ for $72 \mathrm{hrs}$. After 72 hours, fungal balls of mycelia were dislocated from the culture broth, and fungal mycelia were rinsed with sterile water. The collected fungal mass (15 g wet weight) was resuspended in $100 \mathrm{~mL}$ of sterile Milli-Q-Water in a $250 \mathrm{~mL}$ Erlenmeyer flask and then shaken $(150 \mathrm{rpm})$ at $28^{\circ} \mathrm{C}$ for 62 hrs. Next, the cell-free filtrate was collected and added to $\mathrm{Na}_{2} \mathrm{SeO}_{3}$ salt at a concentration of $10 \mathrm{mM}$ (optimum salt concentration from our preliminary experiment). The whole mixture was placed on a shaker $(150 \mathrm{rpm})$ at $28^{\circ} \mathrm{C}$, and the reaction was allowed to occur over $48 \mathrm{hrs}$.

The antimicrobial sensitivity of SeNPs was determined using the agar well diffusion assay (Figure 1, Table 1), and the minimum bactericidal concentration (MBC) was established [9].

\section{Bacterial cell culture and biofilm generation}

Porphyromonas gingivalis ATCC 33277 was able to develop an in vitro mature biofilm on the surface of the Hexacone implant system. The surface was sandblasted, acid-etched during a heat process, and then osmoactively protected. The features of Hexacone implants include an internal 6 or 12-edge, an indoor marginal taper and a US standard internal thread. The implant is composed of sandblasted Ti6AI4V ELI.n (S); each Hexacone fixture was placed in an Eppendorf tube and placed in a sterile air streamline flow cabinet. Each Eppendorf tube was opened and filled with $0.5 \mathrm{~mL}$ of a previously prepared $P$. gingivalis suspension, shaken and then incubated at $37^{\circ} \mathrm{C}$ for 3 weeks.

\section{Microscopic examination}

Sample Preparation: 1-The samples were fixed with $2.5 \%$ glutaraldehyde and dehydrated by serial dilution with ethanol with agitation using an automatic tissue processor (Leica EM TP, Leica Microsystems; Austria). 2- Then, the samples were dried using a CO\% juncture drier (Model: Audosamdri-8 1 5, Tousimis; Rockville, Maryland, USA). 3- The samples were coated by a gold sputter coater (SPI-Module, U).

Microscopic Examination: The samples were investigated by scanning microscopy (JSM-5500 LV; JEOL Ltd. -Japan) under high vacuum at the Regional Center of Mycology and Biotechnology, Cairo, Egypt. Three groups were assigned: one group was P. gingivalis biofilm on Hexacone, the second group was $P$. gingivalis and $25 \mathrm{mg} /$ $\mathrm{ml}$ nanoselenium particles, and the third group was a plank Hexacone fixture. Each group comprised seven SEM microphotographs. The micrographs were processed using Fiji software. 


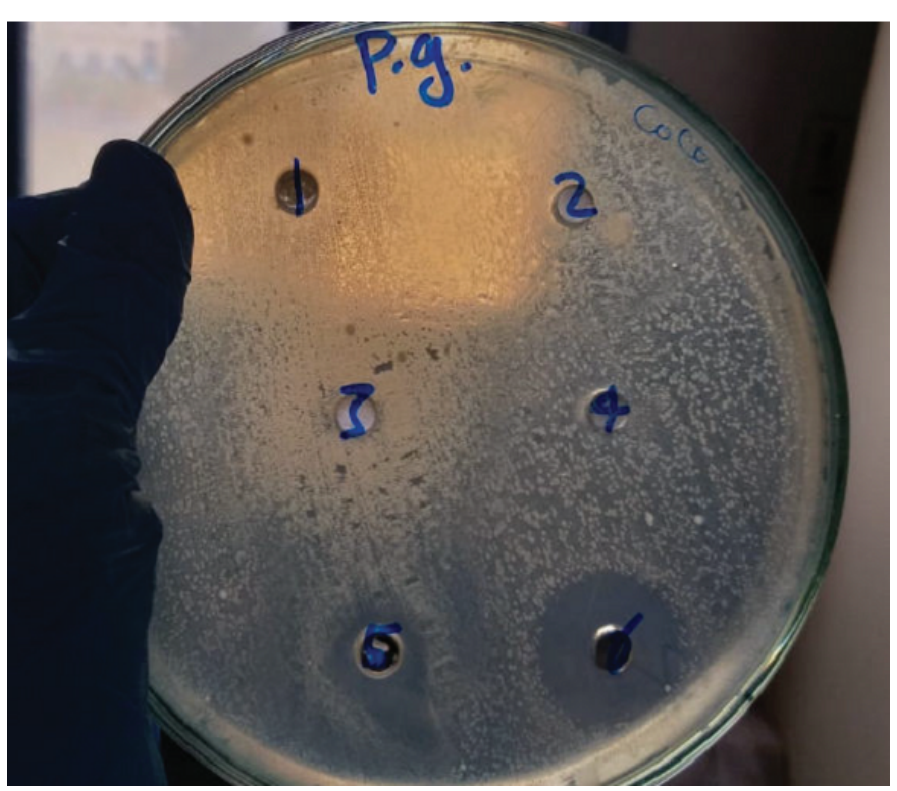

Figure 1: SeNP-sensitive tested isolate.

Table 1: Antimicrobial activity of biogenic SeNPs, mean zone of inhibition in $\mathrm{cm}$ produced on a range of tested pathogenic microorganisms.

\begin{tabular}{|l|c|c|c|c|c|c|}
\hline \multicolumn{7}{|c|}{ Bacterial Isolates $(\mathbf{1 0}$ CFU/ml) } \\
\hline & SeNPs (5 mM) & SeNPs (7 mM) & SeNPs (10 mM) & $\begin{array}{c}\text { T. harzianum cell free water } \\
\text { Extract }\end{array}$ & $\mathbf{N a}_{2} \mathrm{SeO}_{3}$ (7 $\mathbf{~ m M )}$ & Gentamycin \\
\hline $\begin{array}{l}\text { Porphyromonas } \\
\text { gingivalis }\end{array}$ & $4.0 \pm 1.2$ & $5.7 \pm 1.2$ & $4.5 \pm 1.2$ & N.A. & N.A. & 1.6 \\
\hline
\end{tabular}

The test was performed using an agar-well diffusion assay. The well diameter was $6.0 \mathrm{~mm}$ using $100 \mu \mathrm{l}$ of the tested sample.

*RCMB; Regional Center for Mycology and Biotechnology.

**N.A.: No Activity

\section{Visualization of selenium coating and bacterial biofilm}

The dental implants were obtained, and the covered area was calculated with the Fiji image processing freeware (National Institutes of Health, Bethesda, Maryland). The biofilm was segmented from the titanium surface in SEM images using an automatic machine learning process (Figures 2,3). The Trainable Weka Segmentation plugin was utilized to classify the titanium surface, the biofilm and the SeNPs and subsequently segment the image; the information was tabulated and statistically evaluated using SPSS 25 software. Ethics approval was not required for this in vitro study.

\section{Results}

The first group (group 1, P. gingivalis only) and the second group (group 2, P. gingivalis + SeNPs) were compared first. A Shapiro-Wilk test $(\mathrm{P}=0.01$ and $0.04<0.05)$ for both Group 1 and Group 2 (and a visible inspection of their histograms, normal Q-Q plots, $\mathrm{P}-\mathrm{P}$ plots and box plots, the figure showed that the data were not normally distributed for either Group 1 or Group 2. Therefore, the nonparametric MannWhitney test was used rather than the independent t-test.

Effect sizes are reported using the eta square $(\eta 2)$ index from main and interaction effects. Eta square is defined as the proportion of variance within the variable that is explained by the study experimental variable.
Effect Size $=Z / \sqrt{ } N=(2.121) /(\sqrt{ } 7)=80.17 \%$

The results are expressed as the median \pm standard deviation (SD).

To test the hypothesis that Group 1 (mean, SD) and Group 2 means (mean, SD) were equal, a nonparametric Mann-Whitney test was performed. Before conducting the analysis, the normality distribution of every group was examined but was not observed to be satisfied, as mentioned before. The null hypothesis of equal means of every group was rejected, $\mathrm{Z}=2.121, \mathrm{P}<0.0001$. Thus, the Group 1 mean was statistically significantly above the Group 2 mean. The effect size was estimated [31]; the results showed a large effect size of $\eta 2=80.17 \%$, which suggests that $80.17 \%$ of the variance of variable SeNPs was predictable from the 2 groups of $P$. gingivalis when all of the opposite variables were held constant.

The SeNPs affected P. gingivalis biofilms (the effect size was $80.17 \%$ ), and the difference was highly significant ( $\mathrm{p} 0.000$ ), as shown in figure 4 .

\section{Discussion}

This study was the first in vitro study to evaluate the consequences of SeNPs on a major pathogenic cause of periodontitis and periimplantitis, i.e., $P$. gingivalis biofilm formation. As a major etiologic cause of the onset of periodontitis and peri-implantitis [32], the biofilm 


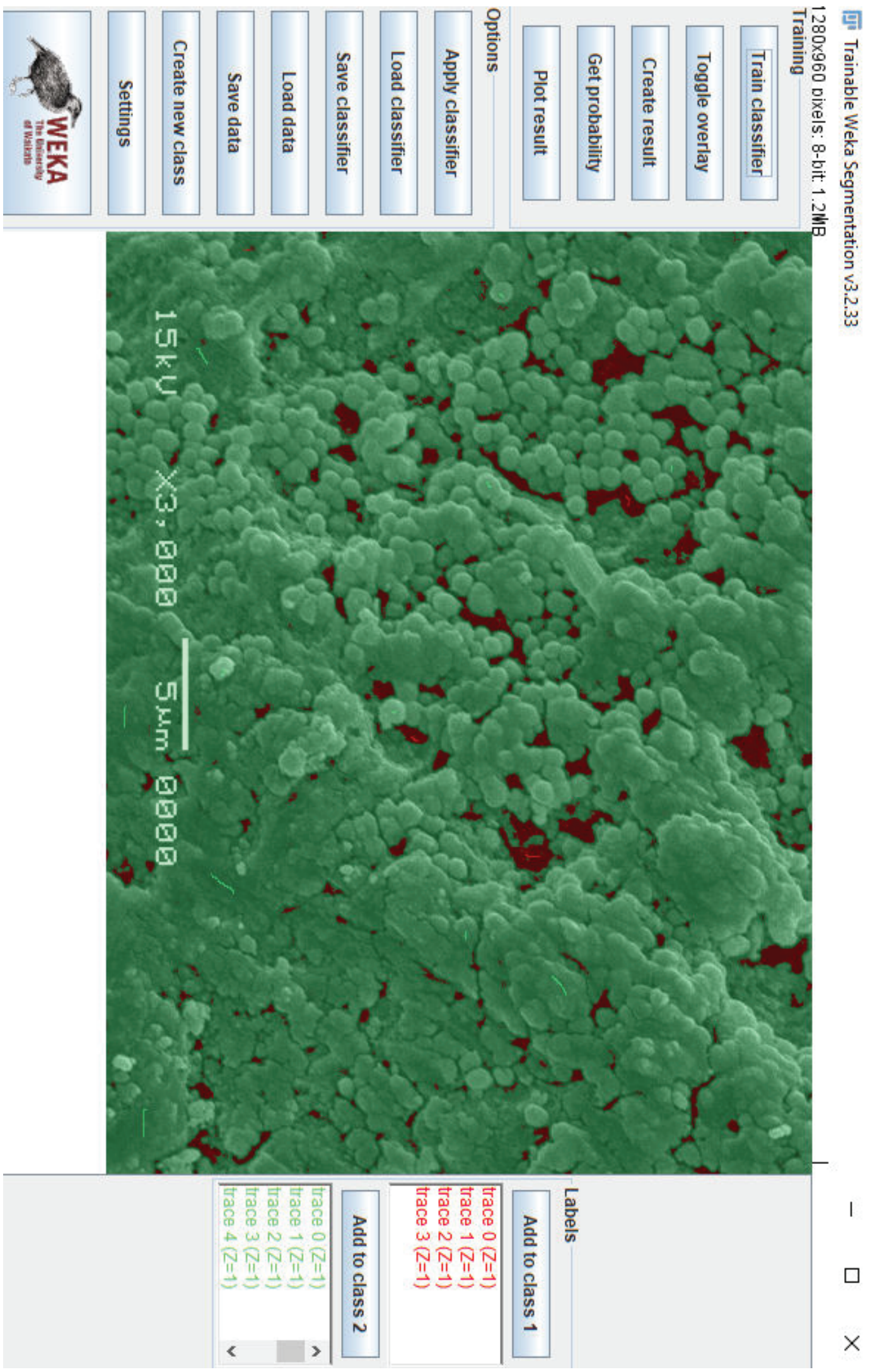

Figure 2: Microphotograph of Porphyromonas gingivalis biofilm without selenium nanoparticles (green denotes the biofilm). The biofilm was segmented from the implant surface by Trainable Weka Segmentation (machine learning software). 


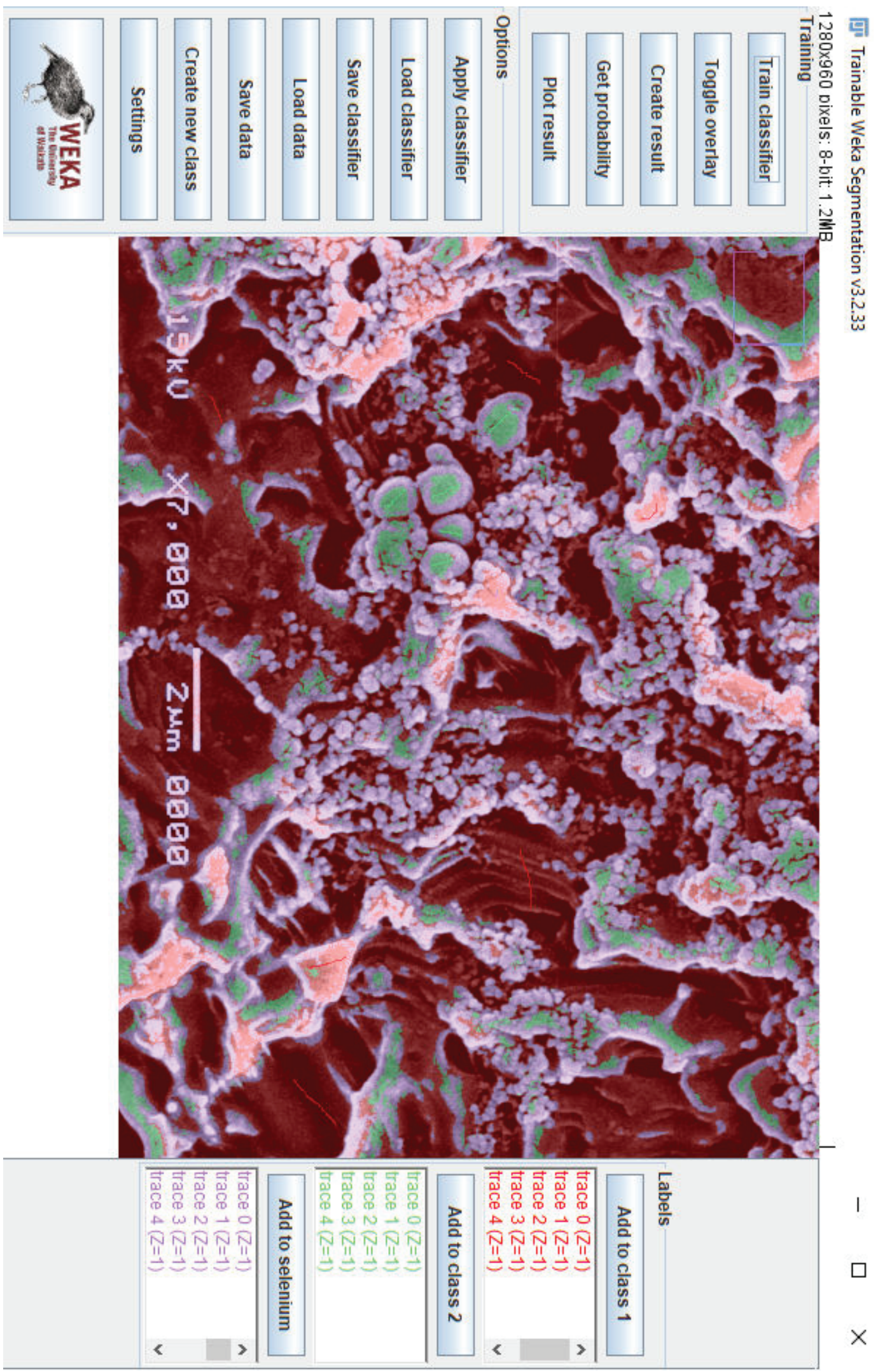

Figure 3: Microphotograph of a P. gingivalis biofilm with selenium nanoparticles (green denotes the biofilm; purple denotes selenium (segmented image). 


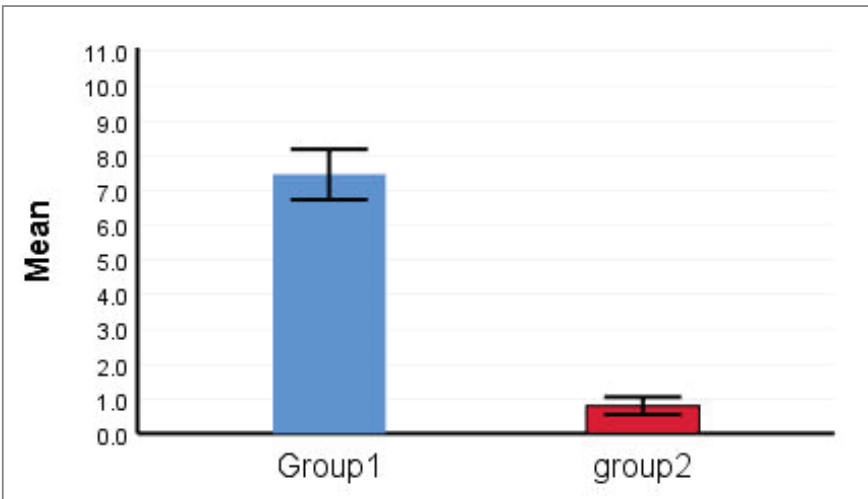

Error Bars: $95 \% \mathrm{Cl}$

Error Bars: +/- $1 \mathrm{SE}$

Figure 4: Bar chart showing the effect size of the SeNPs on Porphyromonas gingivalis biofilm.

is extremely difficult to eliminate because extracellular polymeric substances with polysaccharides shield pathogens from antibacterial agents [33]. This study shows that $25 \mathrm{mg} / \mathrm{ml}$ SeNPs reduced $P$. gingivalis biofilm formation. The inhibitory effect of SeNPs could also be enhanced by increasing the SeNP concentration; the surface should have certain properties to achieve the best rate of osseointegration and combat bacterial challenges, including maintaining host system competence, promoting host tissue integration, inhibiting microbial adhesion and growth and eliminating all organisms on and around the implant [34]. It was also found that $P$. gingivalis proliferation was much lower on $\mathrm{nAg}-\mathrm{HA} / \mathrm{TiO}_{2}$-coated surfaces than on the uncoated controls [35]. It was further found that surface treatment with certain molecules containing antimicrobial and Ti-conjugated peptides may help prevent biofilm formation on Ti surfaces [36]. Chimeric peptides represent a favorable alternative to prevent biofilm formation on titanium surfaces, with the promise of stopping peri-implant disease $[37,38]$.

This consideration is also supported by a study of coCrMo with a $2.5 \mu \mathrm{m}$ zirconium nitride topcoat, which appeared to be a promising surface modification technology that has the ability to inhibit bacterial attachment on the surface of an implant and further prevent implant infection with S. epidermidis biofilm formation [39].

\section{Conclusions}

SeNPs provide a helpful method to inhibit biofilm formation and have prospects in clinical use as antibacterial agents in oral implantology. The use of SeNPs as an implant coating presents promising anti- $P$. gingivalis biofilm activity in vitro and will be further explored for genetic involvement and future clinical applications. The chances regarding local infection control may positively influence the result of implant loss and/or dysfunction. This technology will soon be able to compete in augmenting the clinical success of dental implants.

\section{Authors' Contributions}

Mohamed Assadawy designed and directed the project, prepared the implant, performed image analysis and wrote the paper. Eman Helmy performed microbiological procedures and prepared selenium nanoparticles. Dr. Mohammed Assadawy and Prof Eman Helmy have nothing to disclose.

\section{Consent for Publication}

Not applicable.

\section{Funding}

No funding support for this work.

\section{Ethics Approval and Consent to Participate}

This text does not contain any studies with human candidates or animals accomplished by the author.

\section{Informed Consent}

For this sort of study, formal consent is not required.

\section{Conflict of Interest Statement}

The authors have stated explicitly that there are no conflicts of interest in connection with this article.

\section{References}

1. Wu YT, Willcox M, Zhu H, Stapleton F (2015) Contact lens hygiene compliance and lens case contamination: A review. Cont Lens Anterior Eye 38: 307-316.

2. Vertes A, Hitchins V, Phillips KS (2012) Analytical challenges of microbial biofilms on medical devices. Anal Chem 84: 3858-3866.

3. Peñarrocha-Diago M, Boronat-Lopez A, García-Mira B (2009) Inflammatory implant periapical lesion: etiology, diagnosis, and treatment--presentation of 7 cases. J Oral Maxillofac Surg 67: 168-173.

4. Mahato N, Wu X, Wang L (2016) Management of peri-implantitis: a systematic review, 2010-2015. Springerplus 5: 105.

5. Ata-Ali J, Candel-Marti ME, Flichy-Fernández AJ, Peñarrocha-Oltra D, Balaguer-Martinez JF, et al. (2011) Peri-implantitis: associated microbiota and treatment. Med Oral Patol Oral Cir Bucal 16: e937-e943.

6. Mombelli A, Müller N, Cionca N (2012) The epidemiology of periimplantitis. Clin Oral Implants Res 23: S67-S76.

7. Costerton JW, Stewart PS, Greenberg EP (1999) Bacterial biofilms: a common cause of persistent infections. Science 284: 1318-1322.

8. de la Fuente-Núñez $C$, Reffuveille F, Fernández L, Hancock RE (2013) Bacterial biofilm development as a multicellular adaptation: antibiotic resistance and new therapeutic strategies. Curr Opin Microbiol 16: 580-589.

9. Stewart PS, Costerton JW (2001) Antibiotic resistance of bacteria in biofilms. Lancet 358: 135-138.

10. Davies D (2003) Understanding biofilm resistance to antibacterial agents. Nat Rev Drug Discov 2: 114-122.

11. Holt SC, Kesavalu L, Walker S, Genco CA (1999) Virulence factors of Porphyromonas gingivalis. Periodontol 2000 20: 168-238.

12. Grenier D (1992) Nutritional interactions between two suspected periodontopathogens, Treponema denticola and Porphyromonas gingivalis. Infect Immun 60: 5298-5301.

13. Pihlstrom BL (2001) Periodontal risk assessment, diagnosis and treatment planning. Periodontol 2000 25: 37-58.

14. Zhou Y, Guan X, Zhu W, Liu Z, Wang X, et al. (2014) Capsaicin inhibits Porphyromonas gingivalis growth, biofilm formation, gingivomucosal inflammatory cytokine secretion, and in vitro osteoclastogenesis. Eur J Clin Microbiol Infect Dis 33: 211-219. 
15. Rayman MP (2012) Selenium and human health. Lancet 379: 1256 1268.

16. Wang Q, Webster TJ (2012) Nanostructured selenium for preventing biofilm formation on polycarbonate medical devices. J Biomed Mater Res A 100: 3205-3210.

17. Tran P, Webster TJ (2008) Enhanced osteoblast adhesion on nanostructured selenium compacts for anti-cancer orthopedic applications. Int J Nanomedicine 3: 391-396.

18. Iulian A, Cosmin S, Aurora A (2016) Adhesion aspects in biomaterials and medical devices. J Adhes Sci Technol 30: 1711-1715.

19. El-Ghazaly MA, Fadel N, Rashed E, El-Batal A, Kenawy SA (2016) Antiinflammatory effect of selenium nanoparticles on the inflammation induced in irradiated rats. Can J Physiol Pharmacol 95: 101-110.

20. Cao W, Zhang Y, Wang X, Chen Y, Li Q, et al. (2017) Development of a novel resin-based dental material with dual biocidal modes and sustained release of $\mathrm{Ag}+$ ions based on photocurable core-shell $\mathrm{AgBr} /$ cationic polymer nanocomposites. J Mater Sci Mater Med 28: 103.

21. Song W, Ge S (2019) Application of Antimicrobial Nanoparticles in Dentistry. Molecules 24: 1033.

22. John G, Becker J, Schwarz F (2014) Rotating titanium brush for plaque removal from rough titanium surfaces--an in vitro study. Clin Oral Implants Res 25: 838-842.

23. Park JB, Jang YJ, Choi BK, Kim KK, Ko Y (2013) Treatment with various ultrasonic scaler tips affects efficiency of brushing of SLA titanium discs. J Craniofac Surg 24: e119-e123.

24. Hadi R, Vickery K, Deva A, Charlton T (2010) Biofilm removal by medical device cleaners: comparison of two bioreactor detection assays. J Hosp Infect 74: 160-167.

25. Steffensen SL, Vestergaard MH, Groenning M, Alm M, Franzyk H, et al. (2015) Sustained prevention of biofilm formation on a novel silicone matrix suitable for medical devices. Eur J Pharm Biopharm 94: 305-311.

26. Gomes LC, Mergulhão FJ (2017) SEM Analysis of Surface Impact on Biofilm Antibiotic Treatment. Scanning.

27. Li J, Hirota K, Goto T, Yumoto H, Miyake Y, et al. (2012) Biofilm formation of Candida albicans on implant overdenture materials and its removal. J Dent 40: 686-692.
28. Chan TF, Esedoglu S, Nikolova M (2006) Algorithms for finding global minimizers of image segmentation and denoising models. Siam J Appl Math 66: 1632-1648.

29. Khiralla GM, El-Deeb BA (2015) Antimicrobial and antibiofilm effects of selenium nanoparticles on some foodborne pathogens. LWTFood Sci Technol 63: 1001-1007.

30. Ben-David A, Davidson CE (2014) Estimation method for serial dilution experiments. J Microbiol Methods 107: 214-221.

31. Russell CJ, Bobko P (1992) Moderated regression analysis and Likert scales: Too coarse for comfort. J Appl Psychol 77: 336-342.

32. Lang NP, Berglundh T, Working Group 4 of Seventh European Workshop on Periodontology (2011) Periimplant diseases: where are we now?--Consensus of the Seventh European Workshop on Periodontology. J Clin Periodontol 38: S178-S181.

33. Pöllänen MT, Paino A, Ihalin R (2013) Environmental stimuli shape biofilm formation and the virulence of periodontal pathogens. Int J Mol Sci 14: 17221-17237.

34. Busscher HJ, van der Mei HC, Subbiahdoss G, Jutte PC, van den Dungen JJ, et al. (2012) Biomaterial-associated infection: locating the finish line in the race for the surface. Sci Transl Med.

35. Mo AC, Xu W, Xian S, Li Y, Bai S (2007) Antibacterial activity of silverhydroxyapatite/titania nanocomposite coating on titanium against oral bacteria. Key Engineering Materials, Trans Tech Publications Ltd., Switzerland.

36. Yoshinari M, Kato T, Matsuzaka K, Hayakawa T, Shiba K (2010) Prevention of biofilm formation on titanium surfaces modified with conjugated molecules comprised of antimicrobial and titaniumbinding peptides. Biofouling 26: 103-110.

37. Liu Z, Ma S, Duan S, Xuliang D, Sun Y, et al. (2016) Modification of Titanium Substrates with Chimeric Peptides Comprising Antimicrobial and Titanium-Binding Motifs Connected by Linkers To Inhibit Biofilm Formation. ACS Appl Mater Interfaces 8: 5124-5136.

38. Watson GS, Green DW, Schwarzkopf L, Li X, Cribb BW, et al. (2015) A gecko skin micro/nano structure-A low adhesion, superhydrophobic, anti-wetting, self-cleaning, biocompatible, antibacterial surface. Acta Biomater 21: 109-122.

39. Pilz M, Staats K, Tobudic S, Assadian O, Presterl E, et al. (2019) Zirconium Nitride Coating Reduced Staphylococcus epidermidis Biofilm Formation on Orthopaedic Implant Surfaces: An in vitro study. Clin Orthop Relat Res 477: 461-466. 